\title{
Malignant ureteric obstruction relieved by dexamethasone
}

\author{
THOMAS D. WALSH \\ M.Sc., M.R.C.P.
}

THOMAS S. WEST

St Christopher's Hospice, Sydenham, London SE26 6DZ

\begin{abstract}
Summary
A case is described where dexamethasone relieved tumour-induced ureteric obstruction with improvement in the biochemical abnormalities and clinical features of post-renal failure. Such intervention is not always appropriate but may be of great value in symptom control. Because of this and similar applications, corticosteroids are finding increasing favour in the palliative care of patients with advanced cancer.
\end{abstract}

KEY WORDS: ovarian carcinoma, uraemia, hospice.

\section{Introduction}

Recognized indications for the use of corticosteroids in advanced cancer (Walsh and Saunders, 1984) include raised intracranial pressure, spinal cord compression and hypercalcaemia. During the last decade experience in special palliative care units (Saunders, 1982) has forced a re-examination of traditional practices and prescribing in this area resulting in an extension of the established indications for the use of corticosteroids; we report a case illustrating one of these.

\section{Case report}

A 73-year-old female with ovarian cancer was referred to a hospice for palliative care because of 'terminal' chronic post-renal failure. Oophorectomy (accompanied by subtotal hysterectomy) 2 years previously revealed anaplastic ovarian carcinoma; during surgery the aortic nodes were found to be involved and subsequently a course of chlorambucil was given as well as radiotherapy to the lumbar spine and pelvis for painful bone metastases. Ten days before hospice referral, she was admitted to a general hospital because of an 18-month history of dyspnoea on exertion with recent onset of ankle oedema and episodic dysuria. At that time she was tachypnoeic, blood pressure $150 / 100 \mathrm{mmHg}$ with marked generalized fluid retention. Haemoglobin was $8.6 \mathrm{~g} / \mathrm{dl}$ with normal indices. Biochemical screening showed plasma sodium of $137 \mathrm{mmol} / \mathrm{h}$, bicarbonate 16 mmol/1, urea $21.6 \mathrm{mmol} / 1$, creatinine $507 \mu \mathrm{mol} / 1$. Serum calcium and proteins were normal. Intravenous urography (IVU) showed faint excretion of dye and bilateral pelvicalyceal dilatation, reported as indicating marked parenchymal damage compatible with bilateral obstructive uropathy originating in the pelvis. Treatment was begun with fluid limitation, a restricted protein diet and frusemide $40 \mathrm{mg}$ p.o. daily. Her clinical condition deteriorated progressively, with oliguria and urea rose to $43.2 \mathrm{mmol} / \mathrm{l}$, creatinine to $1309 \mu \mathrm{mol} / \mathrm{l}$, and the potassium to $6 \cdot 6 \mathrm{mmol} / 1$. Because she was severely symptomatic, dexamethasone, $4 \mathrm{mg}$ p.o. qds, was begun in the hope of relieving ureteric obstruction by reduction of periureteric tumour-induced inflammation and oedema. Within $12 \mathrm{hr}$, urine output increased and $48 \mathrm{hr}$ after the dexamethasone was started was $21 / 24 \mathrm{hr}$. Her clinical condition improved markedly with clearing of the confusional state. After 4 days of dexamethasone, the plasma urea had fallen to $20.6 \mathrm{mmol} / 1$. Improvement in her symptoms, general clinical condition and biochemical status was maintained such that, after 14 days, the plasma urea was 16.4 $\mathrm{mmol} / 1$ and bicarbonate was normal. No intravenous fluids or diuretic therapy were used at any time during her hospice admission. The only drugs (other than dexamethasone) given were morphine, prochlorperazine and a laxative. She died 3 weeks after hospice admission; in the intervening time she put her affairs in order (and expressed gratitude for the opportunity to do so) and came to realize, discuss and accept her short prognosis. Post-mortem examination revealed metastatic ovarian adenocarcinoma involving the para-aortic lymph nodes with extensive neoplastic infiltration of retroperitoneal fat on the left side. There was bilateral acute pyelonephritis, bilateral pyohydronephrosis, left-sided extra-ureteric obstruction by tumour just below the hilum and a left intra-renal venous thrombosis. The cause of the right-sided ureteric obstruction demonstrated on IVU could not be decided at post-mortem. 


\section{Discussion}

The pathophysiology of the uraemia was undoubtedly complex but primarily post-renal in origin. Corticosteroids are not thought to be beneficial in acute pyelonephritis and are of no known value in the chemotherapy of ovarian cancer (Cline and Haskell, 1980). Symptomatic benefit followed the improvements in renal function and it is clear that these were due to the introduction of dexamethasone. The clinical history, radiological investigations, sequential biochemical changes and post-mortem findings suggest reduction in periureteric oedema with relief of post-renal obstruction as the basis for the clinical and biochemical improvement produced by the dexamethasone. This case illustrates the beneficial symptomatic effect of corticosteroids when used judiciously to improve quality of life in terminal illness where they are valuable adjunctive agents in symptom control. Controlled studies in this context are often unethical and/or impractical and most therapy empirically based on clinical experience.

Great caution must be exercised in this type of intervention as not only may it be meddlesome (uraemia may be preferable to several other terminal events in carcinoma of the ovary) but each drug has its own adverse effects which may only add to the patient's problems and indeed, once begun, may be difficult to reduce or stop.

\section{References}

Cline, M.J. \& HaSkell, C.M. (1980) Genito-urinary Neoplasms. Cancer Chemotherapy, Vol. 5, 3rd edn., p. 112. W. B. Saunders, Philadelphia.

SAUNDERS, C.M. (1982) Principles of symptom control in terminal care. Medical Clinics of North America, 66, 1169.

WALSH, T.D. \& SAUNDERS, C.M. (1984) Hospice care: the treatment of pain in advanced cancer. Recent Results in Cancer Research. vol. 89, p. 201. Springer-Verlag, Berlin, Heidelberg.

(Accepted 23 March 1983) 